\title{
Fetomaternal Bleeding and Neonatal Hematocrit following Cesarean Delivery: Routine versus Transplacental Transection
}

\author{
Emily Gregory, $\mathrm{MD}^{1} \quad$ Craig V. Towers, MD ${ }^{1} \quad$ Jaclyn van Nes, MD ${ }^{1} \quad$ Kristina Shumard, $\mathrm{MD}^{1}$ \\ Kim B. Fortner, MD ${ }^{1}$ Beth Weitz, WHNP ${ }^{1}$ \\ ${ }^{1}$ Department of Obstetrics \& Gynecology, Maternal-Fetal Medicine, \\ University of Tennessee Medical Center, Knoxville, Tennessee \\ Address for correspondence Craig V. Towers, MD, University of \\ Tennessee Medical Center, 1930 Alcoa Highway, Suite A-435, \\ Knoxville, TN 37920 (e-mail: ctowers@utmck.edu).
} Am J Perinatol Rep 2019;9:e298-e301.

\begin{abstract}
Keywords

- Kleihauer-Betke

- fetomaternal bleeding

- cesarean delivery

- fetomaternal hemorrhage

- neonatal hematocrit

Objective To evaluate fetomaternal bleeding following routine cesarean delivery compared with cesarean delivery involving transplacental transection.

Study Design A prospective cohort study evaluating cesarean delivery in which the placenta was transected (cases) versus controls (placenta not transected) from January 2016 to April 2018. A maternal Kleihauer-Betke's (KB) test and newborn hematocrit were performed shortly after delivery.

Results The rate of a positive KB test was not significantly different between cases $(n=31)$ and controls $(n=61)$ (19 vs. $14 \%$; odds ratio [OR]: $1.44 ; 95 \%$ confidence interval [Cl]: 0.46-4.49; $p=0.74)$. Median neonatal hematocrits were not different. However, the rate of newborn hematocrits $<40 \%$ at delivery was higher in cases compared with controls ( 23 vs. $3 \%$; OR: $8.90 ; 95 \% \mathrm{Cl}: 1.72-45.90 ; p=0.005$ ).

Conclusion A cesarean requiring transplacental transection to accomplish delivery does not significantly increase the rate of fetomaternal bleeding but is associated with a higher likelihood of newborn hematocrit $<40 \%$ at delivery.
\end{abstract}

Fetomaternal hemorrhage has been evaluated in numerous obstetrical clinical conditions, including trauma, amniocentesis, chorionic villus sampling, external cephalic version, first trimester abortion, and bleeding during pregnancy. ${ }^{1-6}$ Fetomaternal bleeding has also been documented following both vaginal and cesarean deliveries, with some studies suggesting an increased risk with cesarean delivery, while others have not found this association. ${ }^{7-9}$ One possible difference in these studies may lie in whether or not the placenta is transected during the cesarean delivery process. Through an extensive literature search using PubMed, Scopus, Medline, and Google Scholar, we found no prior study that has evaluated this question. If fetomaternal bleeding is greater in transplacental transection cesarean deliveries, then Rh-negative mothers who deliver Rh-positive newborns may need supplemental Rh-hyperimmune globulin. In addition, data are also limited regarding neonatal hemat-

received

July 1,2019

accepted

July 9,2019
DOI https://doi.org/

10.1055/s-0039-1697654. ISSN 2157-6998. ocrit postcesarean delivery in procedures where the placenta is transected.

We sought to evaluate the rate of fetomaternal bleeding following routine cesarean delivery compared with cesarean delivery where transplacental transection was needed to accomplish delivery. The secondary study objective was to evaluate neonatal hematocrits postdelivery for each group.

\section{Materials and Methods}

Our study was a prospective cohort evaluation of cesarean delivery controls (the placenta was not transected to accomplish delivery) versus cases (transplacental transection occurred in the delivery process). All pregnant Englishspeaking patients who entered labor and delivery and had a cesarean delivery from January 2016 to April 2018 were eligible. Once consented, a Kleihauer-Betke's (KB) test was
Copyright $\odot 2019$ by Thieme Medical Publishers, Inc., 333 Seventh Avenue, New York, NY 10001, USA. Tel: +1(212) 584-4662.
License terms

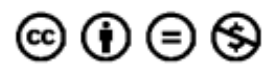


collected within 24 hours of delivery. For laboratory performance, our institution uses Laboratory Corporation of America (LabCorp) and for quantifying fetomaternal hemorrhage, LabCorp uses the KB test. Per LabCorp standardization, a test is considered positive with a cutoff level of $0.05 \%$, suggesting a fetal bleed of $2.5 \mathrm{~mL}$. A neonatal hematocrit was also obtained on the first day of life. Though various normal ranges for neonatal hematocrits have been described, values more than $40 \%$ do not usually require any specific follow-up. A value $<40 \%$ was chosen for our lower level cutoff because at this level further analysis will occur to make sure the level does not decrease to a point where transfusion is needed. Data collection included demographics, gestational age at delivery, indication for cesarean delivery, type of uterine incision, those in labor prior to cesarean, manual removal of the placenta, and whether the placenta was transected to effect delivery of the fetus. Patients with any type of hemoglobinopathy were excluded.

A power analysis was performed based on data from the published studies to date that evaluated the rate of a positive KB test following cesarean delivery. As transplacental transection during cesarean delivery is not a frequent occurrence, a 2:1 ratio of routine cesarean versus transplacental transection cesarean was incorporated. For a power of 80 using an $\alpha$ of 0.05 and an expected rate of a positive KB test to be three times higher in the transplacental transection cases, a minimum of 90 patients was required ( 60 routine cesarean controls and 30 transplacental transection cases). The study progressed until the 30 transplacental transection cases were obtained. Cases number 30 and 31 were obtained on the same day in April 2018. The controls were obtained by November 2017, and no further controls were collected. A large fetomaternal bleed was considered at $15 \mathrm{~mL}$ or greater. As the estimation of the amount of fetal bleeding is not completely exact, to err on the side of safety, many blood banks use this cutoff value to determine if more than one vial of Rh-hyperimmunoglobulin should be administered. Statistics involved the Student's $t$-test, chi-square test, Mann-Whitney's $U$-test, Fisher's exact test, and multivariate analysis where appropriate, and a $p$-value $<0.05$ was considered significant with all tests considered against a two-sided alternative hypothesis. This study was reviewed and approved by the Institutional Review Board of the University of Tennessee Medical Center, Knoxville, TN.

\section{Results}

A total of 94 patients were evaluated with 63 routine cesarean controls compared with 31 transplacental transection cases. The demographics and characteristics of the study population are seen in -Table $\mathbf{1}$, and no differences were found between the cases and controls except for manual removal of the placenta. As depicted, the study population was primarily Caucasian (89\%), multiparous (73\%), and underwent repeat cesarean delivery (69\%).

Overall, there were $15(16 \%)$ positive KB tests (-Table 2) with 9 (14\%, 95\% confidence interval [CI]: $7-25 \%)$ in the routine cesarean controls compared with $6(19 \%, 95 \% \mathrm{CI}$ : $7-31 \%)$ in the 31 transplacental cesarean cases $(p=0.74)$. There were three large bleeds as defined, two in the controls and one in the cases $(p=0.99)$. There was no difference in finding a positive KB test in the multiparous group ( 11 of 69 , $16 \%$ ) versus nulliparous group ( 4 of $25,16 \%$ ), $p=0.99$.

Though there were more instances of manual removal of the placenta in the cases (74\%) versus controls (16\%), this did not affect the rate of a positive KB test. Of the 23 cases, 5 (21.7\%) had a positive KB test compared with 2 of 10 controls (20\%), $p=0.99$. In analyzing all manual removals of the placenta compared with spontaneous placental delivery, again there was no difference in 7 of $33(21.2 \%)$ versus 8 of 61 (13.1\%), $p=0.47$. A multivariate analysis was performed using the variables in -Table $\mathbf{1}$, and none was found to be significant for producing a higher rate of a positive KB test.

Mean neonatal hematocrits were not different $(p=0.17)$. Median neonatal hematocrits with interquartile ranges were also not different with a z-score of $1.08(p=0.28)$. However,

Table 1 Demographics and characteristics of the 94 cesarean delivery study subjects

\begin{tabular}{|l|l|l|l|}
\hline Category & $\begin{array}{l}\text { Cesarean with no transplacental } \\
\text { transection }\end{array}$ & $\begin{array}{l}\text { Transplacental transection } \\
\text { cesarean }\end{array}$ & $p$-Value \\
\hline Number & 63 & 31 & \\
\hline Age (y) & $27.9 \pm 4.9$ & $28.1 \pm 4.8$ & 0.85 \\
\hline Caucasian & $58(92 \%)$ & $26(84 \%)$ & 0.73 \\
\hline Multiparity & $47(75 \%)$ & $22(71 \%)$ & 0.90 \\
\hline Delivery gestational age (wk) & $38.6 \pm 1.9$ & $38.5 \pm 1.8$ & 0.81 \\
\hline Cesarean delivery indication & \multicolumn{2}{|l|}{} \\
\hline Repeat & $45(71 \%)$ & $20(65 \%)$ & 0.66 \\
\hline Breech & $5(8 \%)$ & $2(6 \%)$ & 0.99 \\
\hline Abnormality during labor ${ }^{\text {a }}$ & $13(21 \%)$ & $9(29 \%)$ & 0.52 \\
\hline Classical uterine incision & $2(3 \%)$ & $2(6 \%)$ & 0.60 \\
\hline Manual removal of the placenta & $10(16 \%)$ & $23(74 \%)$ & 0.0001 \\
\hline
\end{tabular}

${ }^{\mathrm{a}}$ Failure to progress, fetal heart monitor indications, or a combination of the two. 
Table 2 A total of 94 cesarean delivery study subjects with 63 routine cesarean deliveries compared with 31 transplacental transection cesarean deliveries

\begin{tabular}{|l|l|l|l|}
\hline Category & $\begin{array}{l}\text { Cesarean with no transplacental } \\
\text { transection }\end{array}$ & $\begin{array}{l}\text { Transplacental transection } \\
\text { cesarean }\end{array}$ & $p$-Value \\
\hline Number & 63 & 31 & $6(19 \%, 95 \% \mathrm{Cl}: 7-31)$ \\
\hline Positive Kleihauer-Betke & $9(14 \%, 95 \% \mathrm{Cl}: 7-25)$ & $1(3 \%, 95 \% \mathrm{Cl}: 0.1-17)$ & 0.74 \\
\hline Large fetomaternal bleed $(>15 \mathrm{~mL})$ & $2(3 \%, 95 \% \mathrm{Cl}: 0.4-11)$ & $48.3 \%( \pm 8.7 \%)$ & 0.17 \\
\hline Mean neonatal hematocrit & $50.4 \%( \pm 5.9 \%)$ & $49.5 \%(\mathrm{QQR}: 40.4-56.5)$ & 0.28 \\
\hline Median neonatal hematocrit & $51.0 \%(\mathrm{IQR}: 46.1-54.5)$ & $32.9-63.4 \%$ & $7(23 \%, 95 \% \mathrm{Cl}: 10-41)$ \\
\hline Neonatal hematocrit range & $36.0-61.6 \%$ & 0.005 \\
\hline Neonatal hematocrit $<40 \%$ & $2(3 \%, 95 \% \mathrm{Cl}: 0.4-11)$ & & 0.96 \\
\hline
\end{tabular}

Abbreviations: $\mathrm{Cl}$, confidence interval; IQR, interquartile range.

for neonatal hematocrits $<40 \%$, there were two in the controls (3\% CI: $0.4-11 \%$ ) compared with seven in the transplacental transection group (23\%, 95\% Cl: $10-41 \%)$, which was significant, $p=0.005$. However, none of these nine newborns required a blood transfusion postdelivery.

\section{Discussion}

This study did not find that a cesarean delivery that requires transplacental transection to accomplish delivery results in a greater risk for fetomaternal bleeding. However, a transplacental transection cesarean delivery did result in an increased risk for a newborn hematocrit to be $<40 \%$ at delivery.

Based on our literature review, this is the first study to analyze whether a transplacental transection cesarean delivery increases the risk for a fetomaternal hemorrhage. Feldman et $\mathrm{l}^{10}$ reported a positive $\mathrm{KB}$ test rate of $18.6 \%$ in 199 cesarean deliveries, which is similar to our rate of $16 \%$. However, their study used a lower KB cutoff value of $1 \mathrm{~mL}$ compared with our cutoff value of $2.5 \mathrm{~mL}$ and most of their study population involved cesarean delivery following labor. These authors also reported a $5 \%$ rate of a fetomaternal bleed of $25 \mathrm{~mL}$ or greater which was similar to our rate of $3 \%$ ( 3 of 94 study participants).

Perslev et al $^{11}$ reported a fetomaternal hemorrhage rate of $18.4 \%$ in 207 elective cesarean deliveries using flow cytometry, but only $1.4 \%$ had a bleed of $5 \mathrm{~mL}$ or greater, which was lower than our rate of $14 \%$ ( 13 of 94 study participants). Lubusky et al $^{7}$ also used flow cytometry to assess fetomaternal hemorrhage and though they found a higher fetomaternal hemorrhage rate in cesarean delivery compared with vaginal delivery, they only reported a $2.3 \%$ rate of a bleed $5 \mathrm{~mL}$ or greater with cesarean delivery.

Adeniji et $\mathrm{al}^{8}$ used the KB test to evaluate the rate of a fetomaternal bleed in 163 deliveries (102 vaginal deliveries and 61 cesarean deliveries). The rate of a positive KB test overall was $10.4 \%$, and the positive $\mathrm{KB}$ test rate between vaginal $(9.8 \%)$ and cesarean delivery $(11.5 \%)$ was not different. They reported a rate for a bleed of $15 \mathrm{~mL}$ or greater in the cesarean delivery group of $7 \%$ ( 4 of 61 ), which was higher but not statistically different from our rate of $3 \%$ (3 of 94), $p=0.43$. None of these studies examined the relationship of transplacental transection at the time of cesarean delivery.
Additionally, in our review, we found no study has reported on the newborn hematocrit following cesarean delivery with transplacental transection. Our study did show a significantly higher rate of a neonatal hematocrit $<40 \%$ in these deliveries. A case of fetal exsanguination with demise was recently reported involving transection of the placenta at the time of cesarean delivery combined with difficulty in fetal head delivery further emphasizing a concern in this clinical setting. ${ }^{12}$

Our study did have a higher positive $K B$ test rate in cesarean deliveries that involved manual removal of the placenta, though the comparison did not reach significance. This lack of significance, however, could represent a type 2 error, and therefore, this is an area that needs further study. Leavitt et $\mathrm{al}^{13}$ demonstrated that draining the fetal blood from the umbilical cord prior to placental delivery did reduce the rate of a positive KB test (using a lower cutoff value of $0.5 \mathrm{~mL}$ of fetal blood) compared with nondrainage, and this could extrapolate somewhat into the potential effect of manual placental removal. None of our deliveries involved draining of the umbilical cord or delayed cord clamping.

Likewise, we only had four cesarean deliveries that involved a classical uterine incision, and therefore, this study cannot answer the effect of this type of uterine entry on the rate of fetomaternal hemorrhage.

The strengths of our study are the prospective study design that controlled for the cesarean indication, type of uterine incision, and manual removal of the placenta, as well as attaining the numbers needed from the power analysis. A study limitation is the potential for a type 2 error. However, if the respective rates of a positive $\mathrm{KB}$ test rate between routine cesarean delivery and transplacental transection cesarean delivery were to remain unchanged, more than 10 times the number of cases and controls would be needed to reach significance. Additionally, as previously discussed, this analysis cannot answer whether manual placental removal or classical uterine incision is risk factors.

Finally, our study used the KB test to evaluate for volume of fetomaternal hemorrhage based on LabCorp protocol. This test is primarily performed because it is inexpensive, requires no special equipment, and is easy to perform in situations where rapid results are needed, which is often the case when 
evaluating for fetomaternal hemorrhage. However, flow cytometry allows for automated results and may be more accurate overall. A few studies have reported on comparisons between the KB test and flow cytometry with most showing flow cytometry to be more accurate; however, the results have not been universal. ${ }^{14-17}$ However, the primary concern with fetomaternal hemorrhage in patients who are Rh-negative would be bleeds that exceed the threshold of one vial of Rhhyperimmune globulin. Both tests appear to be accurate in identifying large fetomaternal bleeds.

\section{Conclusion}

A cesarean requiring transplacental transection to accomplish delivery does not appear to significantly increase the rate of fetomaternal bleeding. However, a transplacental transection cesarean delivery may result in an increased risk for newborn hematocrits to be $<40 \%$ following delivery. Therefore, we recommend that pediatrics be informed in clinical settings where the placenta was transected at the time of cesarean delivery, so these neonates can be evaluated.

\section{Paper Presentation}

Oral presentation, 2018 Annual Meeting, Central Association of Obstetricians Gynecologists, October 17-20, 2018, in Minneapolis, MN.

Funding

This study was funded through the University of Tennessee Physician's Medical Education and Research Foundation.

\section{Disclosure of Interests}

All authors report no conflict of interest and have no financial disclosure to report.

\section{References}

1 Bowman JM. Controversies in Rh prophylaxis. Who needs Rh immune globulin and when should it be given? Am J Obstet Gynecol 1985;151(03):289-294

2 Balderston KD, Towers CV, Rumney PJ, Montgomery D. Is the incidence of fetal-to-maternal hemorrhage increased in patients with third-trimester bleeding? Am J Obstet Gynecol 2003;188 (06):1615-1618, discussion 1618-1621
3 Dhanraj D, Lambers D. The incidences of positive Kleihauer-Betke test in low-risk pregnancies and maternal trauma patients. Am J Obstet Gynecol 2004;190(05):1461-1463

4 Ghidini A, Korker V. Fetal complication after external cephalic version at term: case report and literature review. J Matern Fetal Med 1999;8(04):190-192

5 Katiyar R, Kriplani A, Agarwal N, Bhatla N, Kabra M. Detection of fetomaternal hemorrhage following chorionic villus sampling by Kleihauer Betke test and rise in maternal serum alpha feto protein. Prenat Diagn 2007;27(02):139-142

6 Banerjee K, Kriplani A, Kumar V, Rawat KS, Kabra M. Detecting fetomaternal hemorrhage after first-trimester abortion with the Kleihauer-Betke test and rise in maternal serum alpha-fetoprotein. J Reprod Med 2004;49(03):205-209

7 Lubusky M, Simetka O, Studnickova M, Prochazka M, Ordeltova M, Vomackova K. Fetomaternal hemorrhage in normal vaginal delivery and in delivery by cesarean section. Transfusion 2012;52(09): 1977-1982

8 Adeniji AO, Mabayoje VO, Raji AA, Muhibi MA, Tijani AA, Adeyemi AS. Feto - maternal haemorrhage in parturients: incidence and its determinants. JObstet Gynaecol 2008;28(01):60-63

9 Salim R, Ben-Shlomo I, Nachum Z, Mader R, Shalev E. The incidence of large fetomaternal hemorrhage and the Kleihauer-Betke test. Obstet Gynecol 2005;105(5 Pt 1):1039-1044

10 Feldman N, Skoll A, Sibai B. The incidence of significant fetomaternal hemorrhage in patients undergoing cesarean section. Am J Obstet Gynecol 1990;163(03):855-858

11 Perslev A, Jørgensen FS, Nielsen LK, Berkowicz A, Dziegiel MH. Fetomaternal hemorrhage in women undergoing elective cesarean section. Acta Obstet Gynecol Scand 2011;90(03):253-257

12 Ross MG. Fetal exsanguination from lower segment cesarean section: techniques revisited. JSM Clin Case Rep 2017;5(01): $1126-1127$

13 Leavitt BG, Huff DL, Bell LA, Thurnau GR. Placental drainage of fetal blood at cesarean delivery and feto maternal transfusion: a randomized controlled trial. Obstet Gynecol 2007;110(03): 608-611

14 Fernandes BJ, von Dadelszen P, Fazal I, Bansil N, Ryan G. Flow cytometric assessment of feto-maternal hemorrhage; a comparison with Betke-Kleihauer. Prenat Diagn 2007;27(07):641-643

15 Savithrisowmya S, Singh M, Kriplani A, Agarwal N, Mehra NK, Bhatla $\mathrm{N}$. Assessment of fetomaternal hemorrhage by flow cytometry and Kleihauer-Betke test in Rh-negative pregnancies. Gynecol Obstet Invest 2008;65(02):84-88

16 Meleti D, Caetano AC, Boute T, et al. Assessment of fetomaternal hemorrhage by Kleihauer-Betke test, flow cytometry and $\alpha$-fetoprotein after invasive obstetric procedures. Clin Exp Obstet Gynecol 2012;39(03):303-306

17 Kim YA, Makar RS. Detection of fetomaternal hemorrhage. Am J Hematol 2012;87(04):417-423 